\title{
A Method for Detecting Dynamic Mutation of Complex Systems Using Improved Information Entropy
}

\author{
Bin Ju ${ }^{1,2}{ }^{1}$, Haijiao Zhang ${ }^{1}$, Yongbin Liu ${ }^{1,2, * \mathbb{C}}$, Donghui Pan ${ }^{3}$, Ping Zheng ${ }^{1}$, Lanbing $X u^{1}$ and \\ Guoli $\mathrm{Li}^{1,2}$ \\ 1 College of Electrical Engineering and Automation, Anhui University, Hefei 230601, China; \\ jubin2016@ahu.edu.cn (B.J.); Z16201024@stu.ahu.edu.cn (H.Z.); Z16201019@stu.ahu.edu.cn (P.Z.); \\ xlb97084@ahu.edu.cn (L.X.); liguoli@ahu.edu.cn (G.L.) \\ 2 National Engineering Laboratory of Energy-Saving Motor \& Control Technology, Anhui University, \\ Hefei 230601, China \\ 3 College of Mathematical Sciences, Anhui University, Hefei 230601,China; dhpan@ahu.edu.cn \\ * Correspondence: lyb@ustc.edu.cn
}

Received: 18 December 2018; Accepted: 22 January 2019; Published: 27 January 2019

check for updates

\begin{abstract}
In this study, a nonlinear analysis method called improved information entropy (IIE) is proposed on the basis of constructing a special probability mass function for the normalized analysis of Shannon entropy for a time series. The definition is directly applied to several typical time series, and the characteristic of IIE is analyzed. This method can distinguish different kinds of signals and reflects the complexity of one-dimensional time series of high sensitivity to the changes in signal. Thus, the method is applied to the fault diagnosis of a rolling bearing. Experimental results show that the method can effectively extract the sensitive characteristics of the bearing running state and has fast operation time and minimal parameter requirements.
\end{abstract}

Keywords: nonlinear dynamics; probability mass function; improved information entropy; rolling bearing; fault diagnosis

\section{Introduction}

Mechanical equipment such as a rolling bearing often causes irregular vibrations due to the influence of impacts, variable loads, and friction during operation [1,2]. Therefore, the acquired signals usually have non-stationary and nonlinear characteristics. The bearing is generally analyzed using a combination of various characteristic parameters to evaluate the health of the bearing for the purpose of accurately identifying its state. However, the regularity and sensitivity of the states reflected by various parameters are usually different [3-5]. Meanwhile, the correlation of multiple characteristic parameters often causes difficulties in the analysis. Moreover, using too many feature parameters will affect the computational cost and accuracy of the pattern classification [6]. In addition, achieving satisfactory results is difficult when a traditional linear signal processing method is applied to the feature extraction and recognition of nonlinear signals. The development of nonlinear theory has enabled researchers to propose a series of nonlinear signal processing methods, such as support vector machines [7,8], Kernel Principal Component Analysis [9], Lyapunov exponent [10], symbolic dynamics [11], and Hilbert-Huang transform methods [12,13]. These nonlinear signal processing methods have been used successfully to extract and identify the state characteristics of rolling bearings. These applications have positive importance for exploring new methods of monitoring and fault diagnosis of bearing conditions. 
In recent years, an increasing number of nonlinear analysis methods have been applied to fault diagnosis, such as sample, fuzzy, and multi-scale entropies. The first time that entropy was published for health status monitoring was in 1998 by Basaran and Yan [14]. They utilized the entropy production as the sole measure for evaluating the damage mechanics of solder joints [15]. Sosnovskiy et al. proposed a generalized theory of evolution for mechanothermodynamic systems based on the concept of tribo-fatigue entropy [16]. Yan et al. [17] applied approximate entropy to the health status monitoring of mechanical systems and achieved good monitoring results. Yang et al. [18] combined fuzzy entropy with a variable predictive mode-based class discriminate and applied it to fault diagnosis of rotor systems. Reference [19] applied multi-scale entropy to fault diagnosis of rolling bearings. However, the similarity measure in sample entropy is prone to mutation, and fuzzy entropy calculation is time-consuming; moreover, multi-scale entropy is based on sample entropy [20-22]. These algorithms are greatly affected by the non-stationary nature of time series and are unsuitable for online monitoring. To solve this issue, Bandt et al. [23] proposed a method called permutation entropy (PE) for measuring the complexity of one-dimensional time series. This algorithm features simple calculation, strong anti-noise ability, and short time series and is suitable for online monitoring. The method can effectively detect dynamic catastrophes of complex systems and is widely applied in various fields [24-27]. However, the calculation of PE depends on the selection of embedded dimension $m$ and delay time $\tau$, which affect the effectiveness of PE detection [28].

The occurrence of mechanical faults is a process of mutation. The singularity of signal mutation is important information reflecting mechanical faults. Thus, detecting the signal mutation becomes a key point in studying the operating state of mechanical equipment [29]. The Fourier transform is an effective method to detect signal mutation and is widely applied in various fields [30-32]. However, the Fourier transform method can only determine the overall nature of sudden changes in signal and cannot determine the exact location of these changes in a time domain. With the development of dynamics theory, scholars have proposed a series of methods for detecting signal mutation, such as dynamic transformation regression, nonlinear coherence analysis, and related probability and statistical methods [33-36]. These algorithms can effectively solve the problem but have complex calculation processes and large calculation demands.

To overcome the shortcomings of the existing technology, this study proposes a new method called improved information entropy (IIE) on the basis of constructing a special probability mass function for the normalized analysis of Shannon entropy to measure the complexity of one-dimensional time series. Unlike the PE, the IIE has lesser parameter selection and shorter calculation time. The simulation results show that the IIE can be used as a signal parameter to distinguish different kinds of signals and can effectively detect the mutation in a signal. Therefore, we introduce IIE into the fault signal diagnosis of a bearing. The experimental results show that the IIE can effectively judge the occurrence point and degree of bearing fault.

The rest of the paper is organized as follows. In Section 2, the theoretical background of IIE is introduced, and the characteristics of IIE applied on typical signals are analyzed. In Section 3, two experimental cases are proposed for verifying the effectiveness of the IIE on the fault diagnosis of rolling bearings. In Section 4, the conclusions are elaborated.

\section{Methodology}

\subsection{Basic Definition of the Proposed Improved Information Entropy}

A set of time series composed of $N$ points is considered, and these variables can be written as $\left\{x_{i}, i=1,2,3, \cdots, N\right\}$. The Shannon entropy can be used to quantify the uncertainty of the given time series. In this calculation of the entropy, the extraction of the probability mass function (PMF) is an essential step. In fact, if there are some outliers or mutations in a given time series, they will deviate greatly from the mean of the sequence, and the probability of occurrence is very low. In order to 
highlight and evaluate the effect of the deviation degree on the entropy calculation, we construct the following PMF $p(i)$ for each data point $x_{i}$ :

$$
p(i)=e^{-\frac{\left(x_{i}-\mu\right)^{2}}{2 \sigma^{2}}}, i=1,2, \cdots, N
$$

where $\mu$ and $\sigma$ are the mean and standard deviations of the time series, respectively, and can be calculated as follows:

$$
\begin{gathered}
\mu=\frac{1}{N} \sum_{i=1}^{N} x_{i}, \\
\sigma=\sqrt{\frac{1}{N-1} \sum_{i=1}^{N}\left(x_{i}-\mu\right)^{2} .}
\end{gathered}
$$

It is worth noting that the PMF $p(i)$ constructed in Equation (1) has the following characteristics:

(1) $0 \leq p(i) \leq 1$.

(2) $p(i)$ will approximate to 1 when $x_{i}$ is close to $\mu$, and then $-\ln p(i)$ will approach 0 .

(3) $p(i)$ will approximate to 0 when $x_{i}$ is away from $\mu$, and then $-\ln p(i)$ will approach infinity.

It is necessary to construct such PMF $p(i)$ for the following calculation of the entropy to reflect the mutation characteristics of a time series. If there occurs an outlier or a mutation $x_{i}$ in the time series, it is far away from the mean of the sequence. In this case, the $p(i)$ will be close to 0 , using the aforementioned constructed PMF. Therefore, the effect of the mutation will be amplified in the calculation of the entropy. The Shannon entropy $H$ is a non-negative index to measure the complexity of time series, and can be calculated as follows:

$$
H=-\sum_{i=1}^{N} p(i) \ln (p(i))
$$

By normalizing the entropy $H$ above, a parameter of improved information entropy (IIE) is defined to quickly detect the dynamic mutation of one-dimensional time series. The IIE is given by

$$
\mathrm{IIE}=-\ln \left(\frac{H}{N}\right)
$$

where $N$ is the length of the time series mentioned above.

This normalization processing is done in order to make the calculated entropy weakly correlated with the length $N$ since the parameter of $N$ directly influences the calculation speed. So next, we verify the effect of the data length $N$ on IIE below. As shown in Figure 1, the entropy values of $H$ and IIE calculations are both performed on sine signals, square wave signals, and white noise of lengths of $100,200,300, \ldots, 4000$. According to the calculation results, the values of $H$ increase with the length $N$, which is not suitable for the entropy comparison for different signals with different lengths. Whereas, after the normalization process, the calculated entropy values of IIE weakly correlate with the length $N$ as shown in Figure $1 \mathrm{~b}$. The results show that the three signals fluctuate at the beginning because the initial time series is too short and the randomness of the signals is insufficiently stable. However, as the time series length increases, the IIE values of the three signals are stable near a constant value. Therefore, the IIE has less dependence on the length of the time series. For the difference in the IIE values of different kinds of signals, a detailed analysis will be carried out in the next section. 


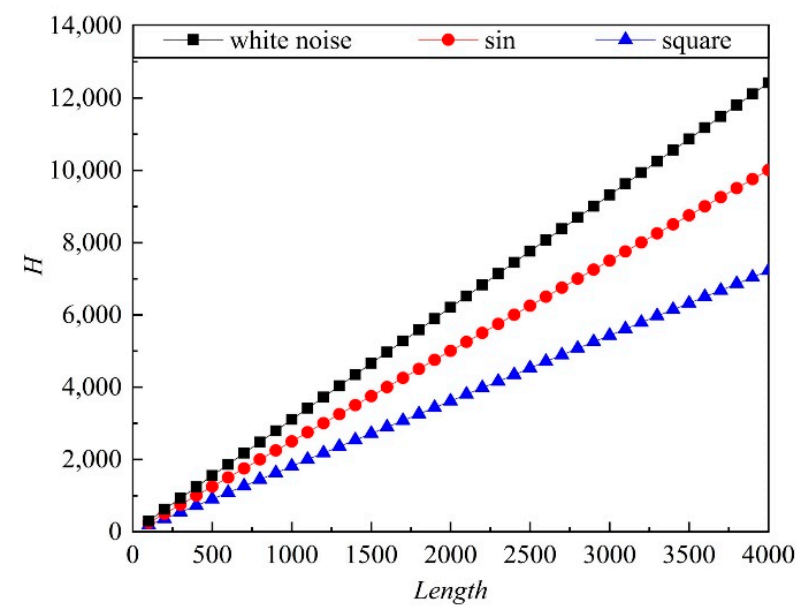

(a)

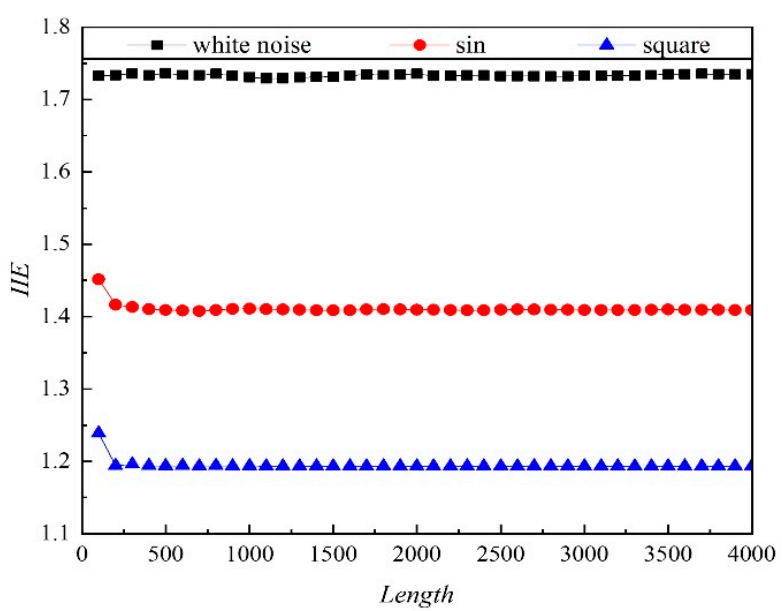

(b)

Figure 1. The effect of data length on the entropy values of $H$ (a) and improved information entropy (IIE) (b) of different signals.

\subsection{The IIE Characteristics of Typical Signals}

On the basis of the definition of the IIE above, we calculate the IIE of several typical signals, as shown in Figure 2. These typical signals include a sine wave, square wave, white noise, $1 / \mathrm{f}$ noise, and sine signal superimposed with different noise signals. Each calculated IIE value corresponds to a time series length of 2048. For a single signal (sine wave, square wave, white noise, and $1 / \mathrm{f}$ noise), we consider it as a noise signal and calculate the IIE value under different signal intensities. For sine signals superimposed with different noise signals, the intensity of the sine signal is set to $17 \mathrm{~dB}$, and the IIE variation with superimposed noise under different intensities is calculated.

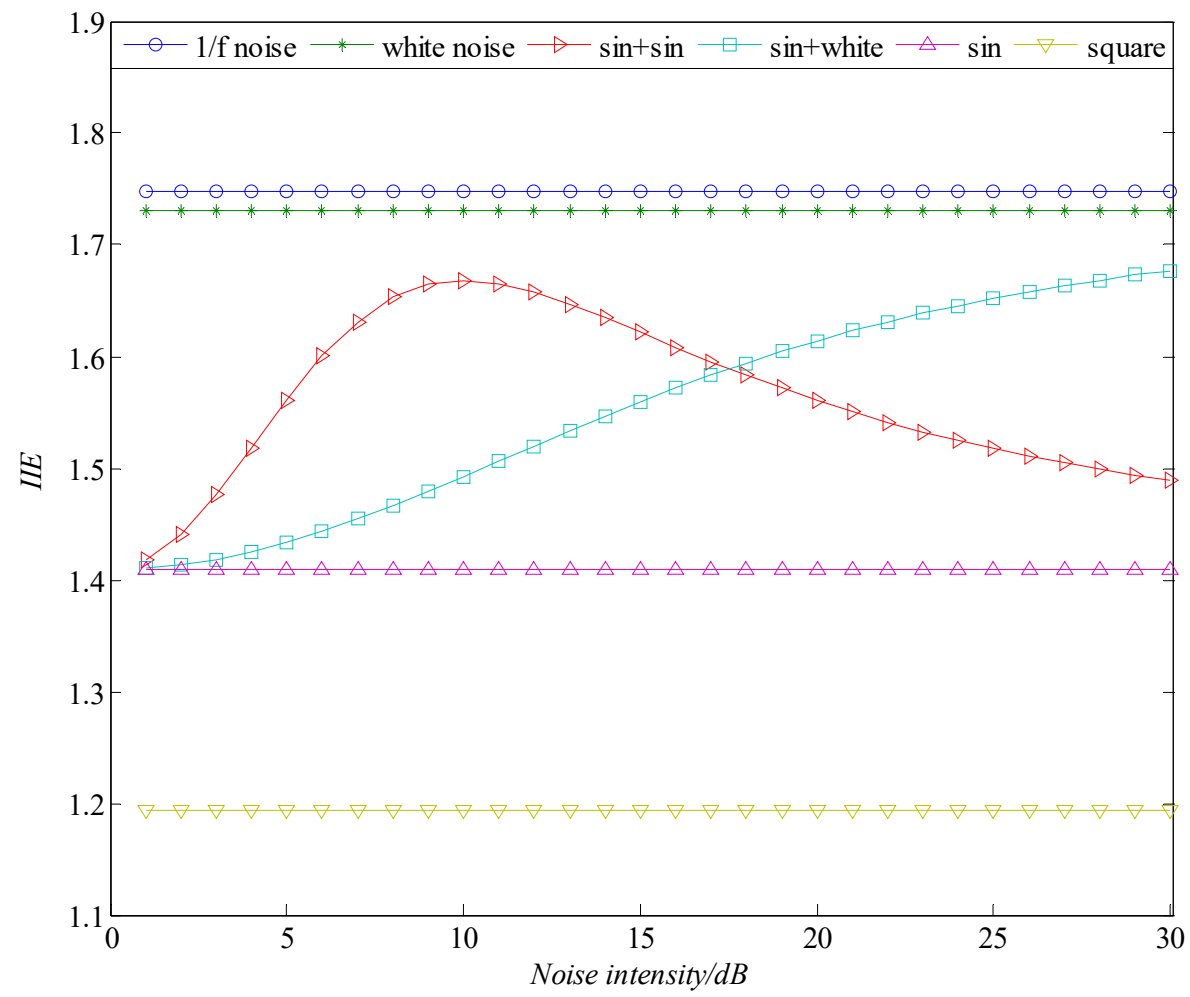

Figure 2. Variation in IIE of several typical signals with different noise intensities. 
As shown in Figure 2, the entropy value for a single signal is very stable with the increase in the signal intensity. This result is due to the fact that the degree of randomness is definite for a certain single signal; the noise signal has the largest degree of randomness, followed by the sine wave, whereas the square signal has the smallest degree of randomness. The randomness can also be reflected by the IIE values of these single signals; the entropy value of the square wave is the smallest, the sine wave is in the middle, and the noise signal is the largest. Thus, the IIE can be a characteristic parameter that reflects the degree of randomness of a time series and has a certain degree of discrimination for different signals. A high value of entropy indicates high randomness of the signal and vice versa. The IIE values of broadband and narrowband noise signals are very high and have little difference compared with those of the white noise and $1 / \mathrm{f}$ noise. The randomness of the two noise signals is large but inconsiderably differ. Therefore, only white noise is selected when calculating the IIE value of the sine signal superimposed with different noises. In the actual test environment, some regular noise signals may be coupled. Thus, the IIE analysis of sine signal superimposed with sine noise is also conducted.

Figure 2 shows the IIE values of a sine signal superimposed with sine and white noises of different intensities. The amount of information contained in the signal increases due to the superposition of different signals. As a result, the value of entropy is higher than that of a single signal. When the noise intensity reaches a certain value, the noise signal will gradually cover the sine signal, and the value of entropy will gradually approach that of the noise signal. Thus, the IIE curve in which the sine signal is superimposed with white noise tends to rise gradually. With the increase in noise intensity, the IIE value of the sine signal superimposed with sine noise increases first and then decreases. This phenomenon occurs because, when the intensity of sine noise is lower than that of the sine signal, the randomness is enhanced after the superposition. When the intensity of sine noise exceeds that of sine signal, the sine noise dominates, and the value of entropy approaches that of the sine noise gradually; that is, it begins to decline.

\subsection{Dynamics Mutation Simulation of Time Series Based on IIE}

In this section, we test whether the IIE algorithm is reasonable for detecting the dynamic mutation in vibration signal analysis. First, a set of simulation signals is presented. The simulation signal is a set of sinusoidal signals, which is superimposed with a white noise signal at the times of $0-10 \mathrm{~s}$ and 20-30 s, respectively, and is shown in Figure 3a. Then, the PE and IIE of the signal are calculated. When calculating the PE, the parameter of the embedded dimension is $m=5$ and the time delay is $\tau=2$ [37]. The computational results of the two entropies are shown in Figure 3b,c. Figure $3 c$ shows that, after the noise signal is superimposed on the sinusoidal signal at the time slice of $0-10 \mathrm{~s}$, the IIE is very large and approaches 1.8, and the volatility is small. This finding indicates that the signal is in a completely random state. When the noise disappears, the signal IIE decreases immediately. At the mutation point of the signal, the IIE jumps. At the mutation point of time $20 \mathrm{~s}$, the noise is superimposed on the original signal again. The signal returns to a disordered state, and the IIE rises immediately to a high value and stabilizes. The PE value exhibits the same variation trend of the signal as the IIE does, as shown in Figure 3b. Thus, if the signal for a given random time series has a sudden change or changes the original state, then the IIE value will show an evident mutation at the corresponding time point. Therefore, the signal simulation analysis demonstrates that the IIE algorithm can detect mutations and perform state recognition. This characteristic of the IIE algorithm allows for its utilization in signal analyses of fault diagnosis. 


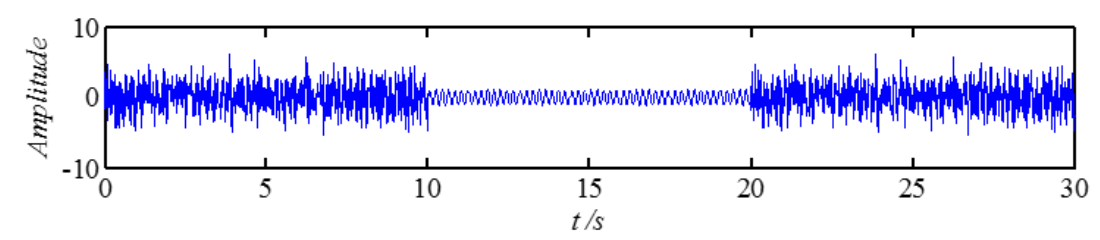

(a)

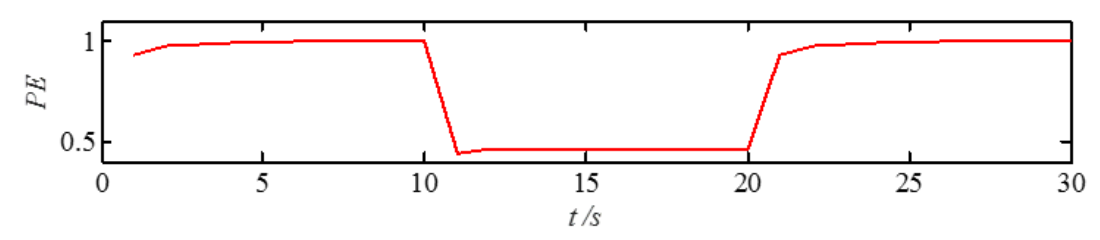

(b)

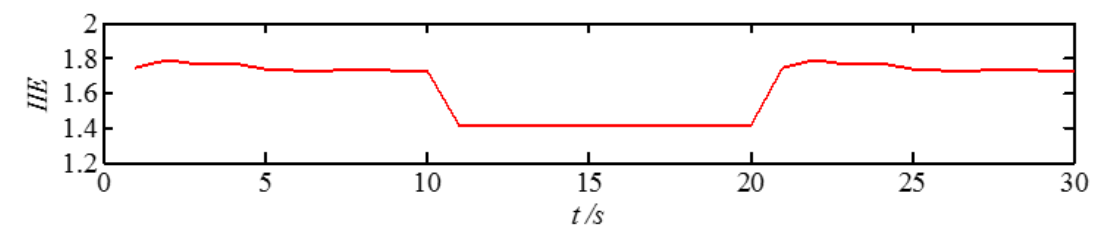

(c)

Figure 3. Entropy change chart of the simulation signal. (a) original signal; (b) permutation entropy (PE); (c) improved information entropy (IIE).

\subsection{Running Time of the IIE Compared with that of the PE}

The PE and IIE are analyzed in terms of running time. In calculating PE, we need to consider and set three parameters, namely, the length of time series $N$, embedded dimension $m$, and time delay $\tau$. Moreover, the determination of $m$ and $\tau$ still depends on experience and on the attempt, which is a bottleneck problem for the PE in engineering applications. However, in calculating IIE, we only need to consider the length of time series $N$. The effect of embedding dimension and time delay on runtime in PE is illustrated in Table 1, where the PE is calculated by selecting dimension $m=1-7$ under time delay $\tau=1-4$. Table 1 shows that, when less embedded dimension and time delay are used, the running time is short. However, in the practical application of mechanical vibration signals, the validity of calculation results will be affected. Cao et al. emphasized that, when $m<4$, the signal mutation cannot be accurately detected; when $m>7$, the reconstructed phase space will homogenize the signal, and abrupt changes in the signal can be detected effectively but with difficulty; when $\tau>5$, small changes in the signal cannot be detected [37]. If the parameter selection is too small or too large, then the validity of the calculation result will be affected. Moreover, if the parameter selection is too large, then the calculation amount will also be large.

Table 1. Running time under different embedded dimensions and time delays.

\begin{tabular}{cccccccc}
\hline $\boldsymbol{\tau} \backslash \boldsymbol{m}$ & $\mathbf{1}$ & $\mathbf{2}$ & $\mathbf{3}$ & $\mathbf{4}$ & $\mathbf{5}$ & $\mathbf{6}$ & $\mathbf{7}$ \\
\hline 1 & 0.008 & 0.008 & 0.009 & 0.023 & 0.093 & 0.518 & 3.615 \\
2 & 0.003 & 0.006 & 0.009 & 0.022 & 0.090 & 0.512 & 3.515 \\
3 & 0.003 & 0.006 & 0.009 & 0.022 & 0.088 & 0.510 & 3.545 \\
4 & 0.003 & 0.006 & 0.009 & 0.022 & 0.088 & 0.533 & 3.432 \\
\hline
\end{tabular}

A Gaussian white noise signal with a noise length of 1000 and a noise intensity of $10 \mathrm{~dB}$ are used to calculate the running time of PE and IIE separately. Specifically, the PE has embedded dimension $m=5$ and time delay $\tau=2$. The computation cost of PE is approximately $0.090 \mathrm{~s}$, and the computation cost of IIE is nearly $0.028 \mathrm{~s}$. The algorithms are run using the MATLAB R2014b (MATLAB 8.4, The Mathworks, 
Inc., Natick, MA, USA) platform on a laptop with a $3.2 \mathrm{GHz}$ AMD Ryzen 51400 CPU and 4.0 GB RAM. The calculation results show that the running time of IIE is shorter than that of PE.

\section{Application Cases Using IIE for Defect Diagnosis of Bearings}

\subsection{Rolling Bearing Fault Diagnosis Based on IIE}

If the fault signal is weak during rolling bearing failure, then it is easily covered by other signals (noise). Therefore, extracting feature vectors from fault signals is particularly critical. During the operation of the bearing, the vibration signal easily exhibits non-stationary and nonlinear characteristics. Besides, in the working process of the bearing, the vibration signal may couple with its own nonlinear factors due to the sudden situation and the randomness. In practice, the vibration signal received by the signal acquisition device is usually a one-dimensional time series. When the fault point of the rolling bearing occurs in different parts, the vibration trend of the one-dimensional vector is different. This characteristic can be utilized to trace the health state of the bearing system. Therefore, the IIE algorithm proposed above for rapid detection of vibration signal saltation is considered to be applied in bearing fault diagnosis experiments.

The computational flowchart of IIE is shown in Figure 4. Overall, the bearing fault diagnosis based on the IIE algorithm can be summarized as follows:

(1) Acquire data.

(2) Constructing time series: The vibration signals collected from rolling bearings are constructed into time series $x_{i}$.

(3) Calculating the $\mu$ and $\sigma$ of time series $x_{i}$.

(4) Constructing the PMF $p(i)$.

(5) Computing the Shannon entropy $H$.

(6) Obtaining the parameter of IIE by normalizing $H$ with data length $N$.

(7) Determining the state of the system.

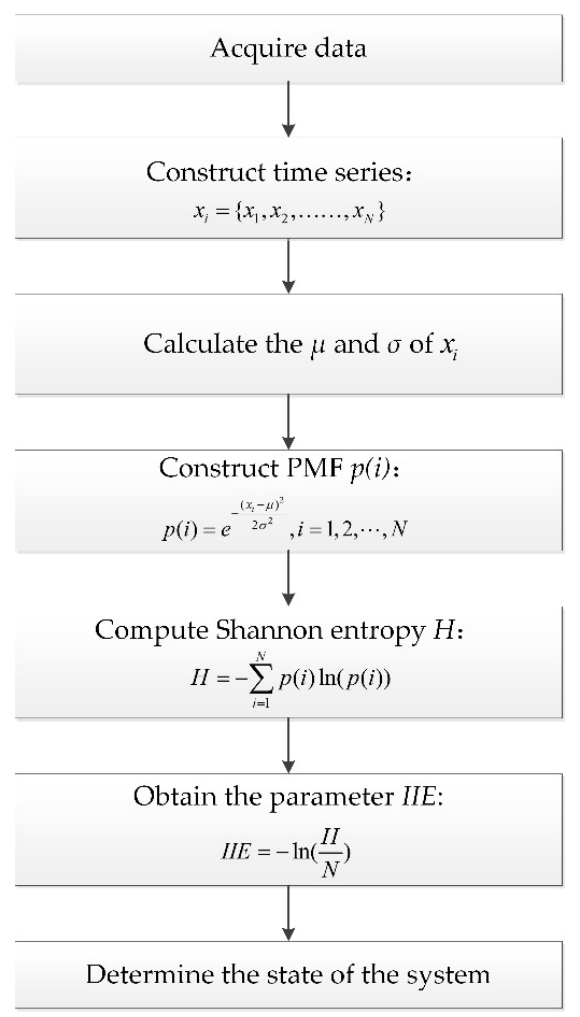

Figure 4. Flowchart of bearing fault diagnosis based on IIE. 
In accordance with the results of the IIE analysis, the state of the system operation is judged.

\subsection{Experimental Result Analysis}

Case I: To verify the effectiveness of the proposed method, the experiment is implemented for the bearing vibration signal of the whole lifecycle; all data sets are obtained from the Intelligent Maintenance System Center [38]. The test platform is shown in Figure 5. In the test system, four Rexnord ZA-2115 bearings (Rexnord Corporation, Milwaukee, WI, USA) are mounted on the same test shaft. They are rotated at $2000 \mathrm{rpm}$ by an alternating current motor. Then, a radial load of 6000 pounds is added to the bearing. Four PCB 353B33 accelerometers (PCB Piezotronics, Inc., Buffalo, NY, USA) are installed on the bearing to collect vibration signals. The sampling rate and data length are set to $20 \mathrm{kHz}$ and 20,480 points, respectively. Simultaneously, the NI DAQ card -6062E data acquisition card is used to record the vibration signal every $10 \mathrm{~min}$. Magnetic screw plugs are installed in the oil feedback pipeline. As the bearings wear away, metal debris is gradually absorbed on the magnetic screw plugs. After exceeding a certain level, the machine stops running. At the end of the test, inner race, roller element, and outer race defects occur in bearings 3 and 4 of test 1 and bearing 1 of test 2 . Bearing parts with different defects are shown in Figure 6. Additional details of the experiment can be found in Reference [39].

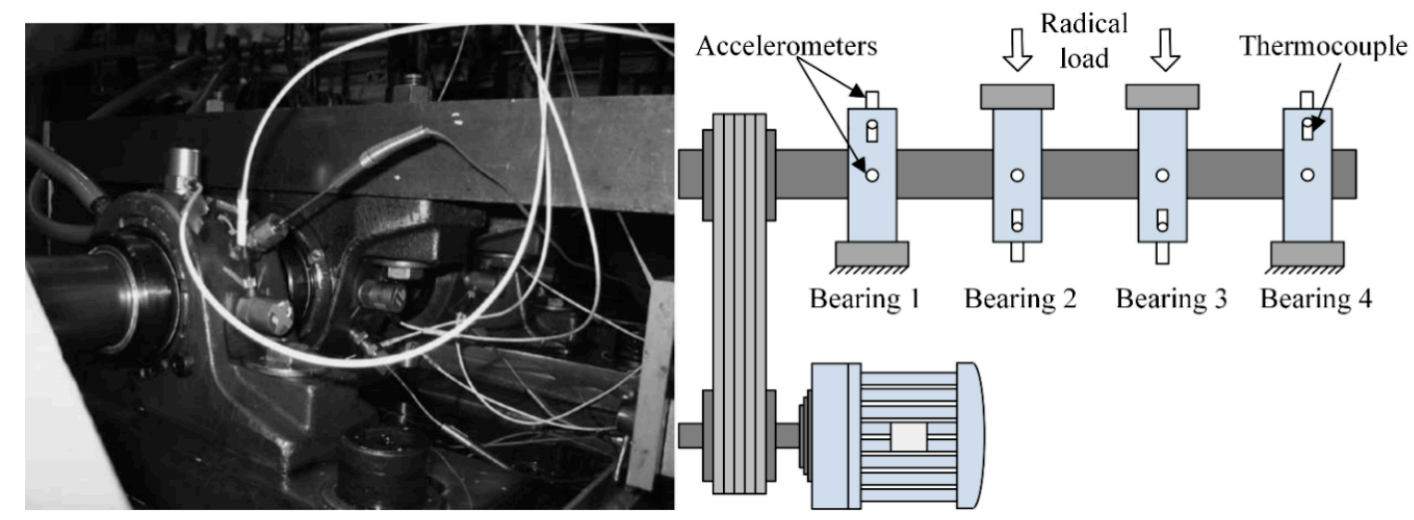

Figure 5. The bearing test platform.
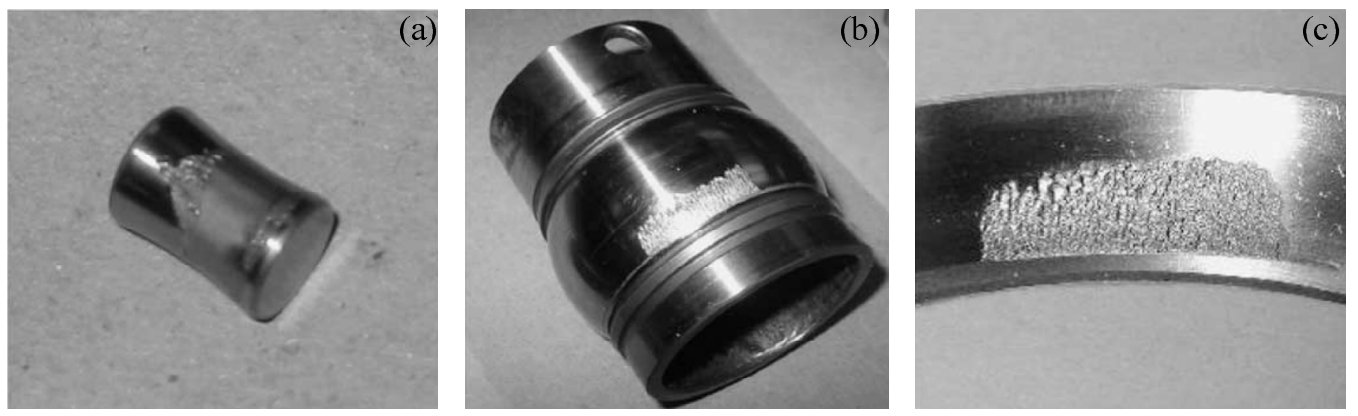

Figure 6. The tested bearing with different fault types: (a) Roller element defect, (b) inner race defect, and (c) outer race defect.

Figure 7 depicts the IIE trend charts of the whole lifecycle signals with different bearing failures. As shown in Figure 7a, the inner race of the bearing is relatively stable before $136 \mathrm{~h}$. When the bearing runs to $136 \mathrm{~h}$, its vibration signal shows a slight jump. This condition indicates that the bearing has begun to appear abnormal. From 136-160 h, the vibration signal fluctuates up and down. Therefore, the bearing is running with a fault, but the fault is not serious. When the bearing runs for more than $160 \mathrm{~h}$, its vibration signal changes drastically, and its entropy value reaches the maximum in $163.3 \mathrm{~h}$. At this time, the bearing has experienced serious failure and reached its life limit. As shown in Figure $7 \mathrm{~b}$, the outer race bearing is relatively stable before $108 \mathrm{~h}$. When the bearing runs to $108 \mathrm{~h}$, its vibration 
signal shows a slight jump. This condition indicates that the bearing has begun to appear abnormal. From 108-160 h, the vibration signal fluctuates up and down. Therefore, the bearing is running with a fault, but the fault is not serious. When the bearing runs for more than $160 \mathrm{~h}$, its vibration signal changes drastically, and its entropy value reaches the maximum in $163.3 \mathrm{~h}$. At this time, the bearing has experienced serious failure and reached its life limit. The whole life analysis diagram of the roller element is shown in Figure 7c. The graph shows that the fluctuation trend between 10-45 $\mathrm{h}$ and $110-160 \mathrm{~h}$ can be explained by the nature of the damage propagation process. When operating between 10 and $45 \mathrm{~h}$, the roller element surface defect has begun, thereby forming a small flake or crack and then gradually smoothing by continuous rolling contact. From 110-160 h, the roller element surface defect extends to a wide range, and the vibration level rises again. At this time, the bearing has experienced a serious fault to reach its life limit. This phenomenon of "healing" can be confirmed in Reference [39].

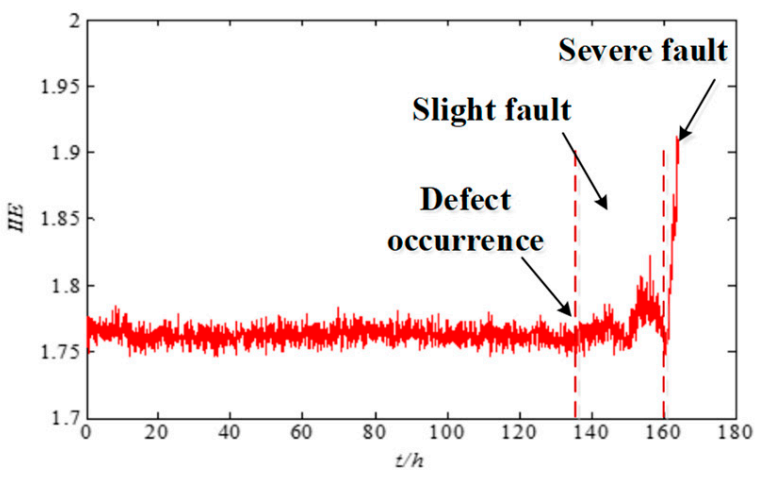

(a)

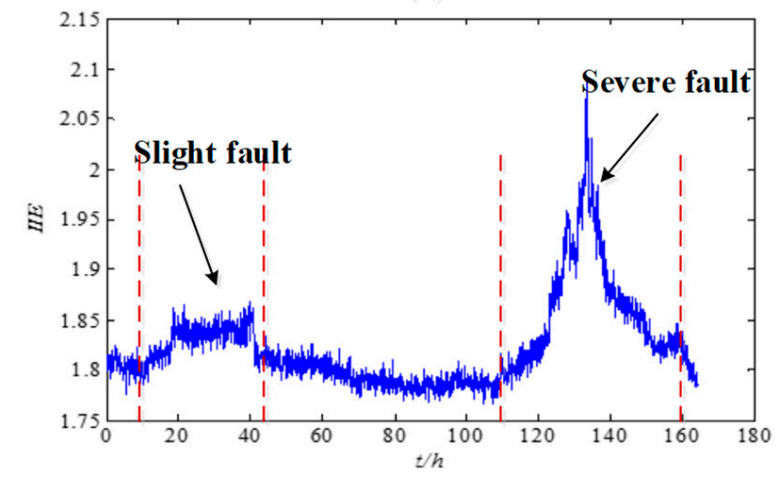

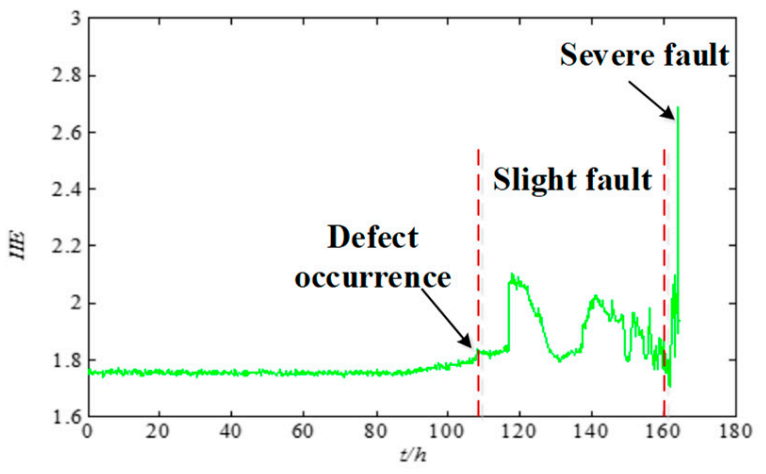

(b)

(c)

Figure 7. The IIE trend charts of vibration signals from the NASA website: (a) Inner race, (b) outer race, and (c) roller element.

Case II: Apart from the IIE extraction and analysis of the faulty bearing data provided by the NASA website, this study also analyzes the bearing life data obtained by our laboratory. The experimental platform, as shown in Figure 8, includes ABLT test platform, signal amplifier, monitoring system, four tested bearings, NI PXI acquisition system, and signal acquisition program based on LabVIEW 2013 (National Instruments Corporation, Austin, TX, USA). The ABLT test platform comes from Hangzhou Bearing Test and Research Center and is composed of but not limited to a transmission system, load system, and lubrication system. The bearings used in the experiment are HRB 6305 (Harbin bearing manufacturing company, Harbin, China). They are mounted on the same experimental shaft and connected to the motor through a belt, which is powered by a three-phase motor. During the operation, the bearings are added with a radial load of $750 \mathrm{~kg}$ to hasten the defect occurrence. The rotating speed of the shaft and the sampling frequency are set as $3000 \mathrm{rpm}$ and $20 \mathrm{kHz}$, respectively. The vibration signals of each bearing are acquired every $5 \mathrm{~min}$ using the LABVIEW 
acquisition program. After a long period of fatigue tests, different types of bearing failures have been obtained, as shown in Figure 9.

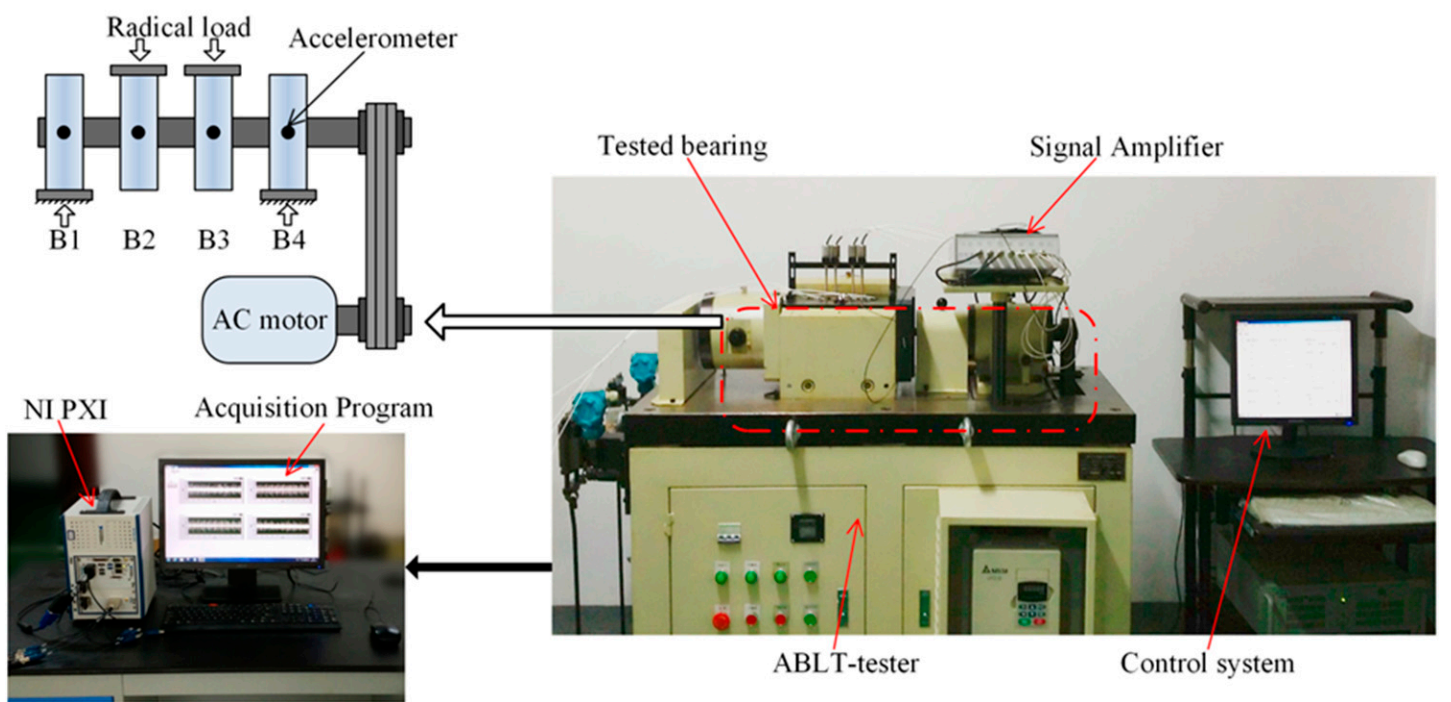

Figure 8. Designed bearing test platform.

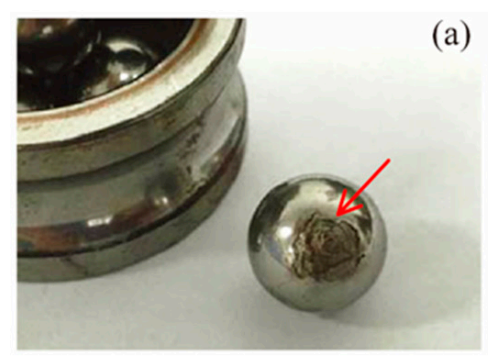

(a)

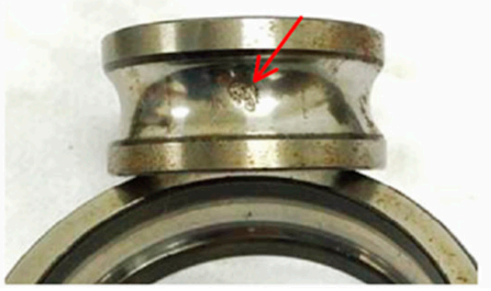

(c)

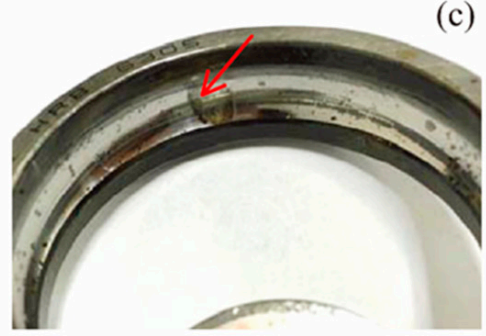

Figure 9. The tested bearing with different fault types: (a) Roller element defect, (b) inner race defect, and (c) outer race defect.

The acquired experimental data are analyzed to verify the proposed IIE feature extraction method for the fault diagnosis of the bearing. The fault vibration signals of the bearing inner ring, bearing outer ring, and rolling element are usually used as analysis objects in validating bearing performance degradation. As shown in Figure 9, the bearing runs continuously for more than $40 \mathrm{~h}$ by applying a radial load to accelerate its fatigue failure. Calculation results of the IIE of the whole life vibration signals of bearing in Figure 10a-c show that the bearing performance degradation curves of the inner race, outer race, and roller element extracted from IIE model have good monotonicity and stable change trends. The whole lifecycle of the bearings can be clearly shown from healthy status to minor failures and the serious failures of the degradation process. Therefore, the proposed IIE can be used for performance degradation indicator extraction. This method is sensitive for early fault detection, which is crucial for setting an early alarm for timely maintenance and prevention of cascading failure. 


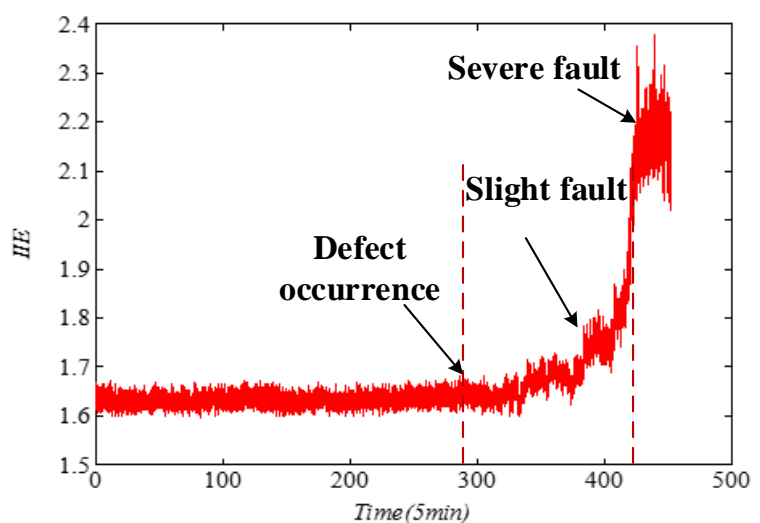

(a)

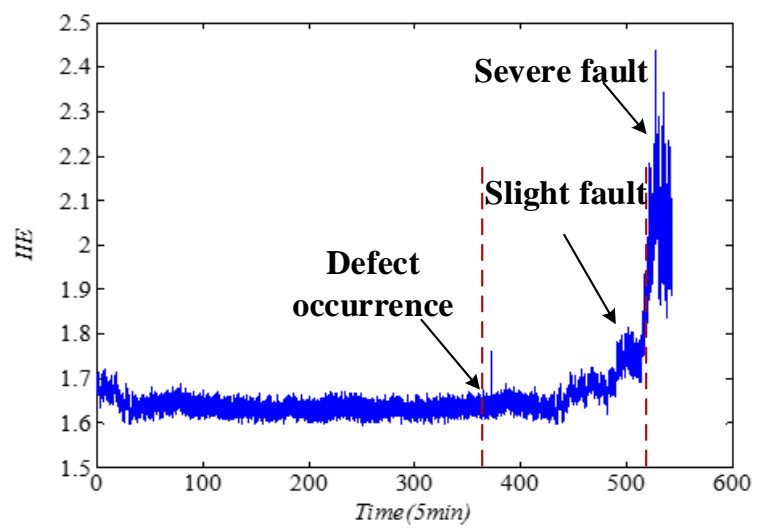

(c)

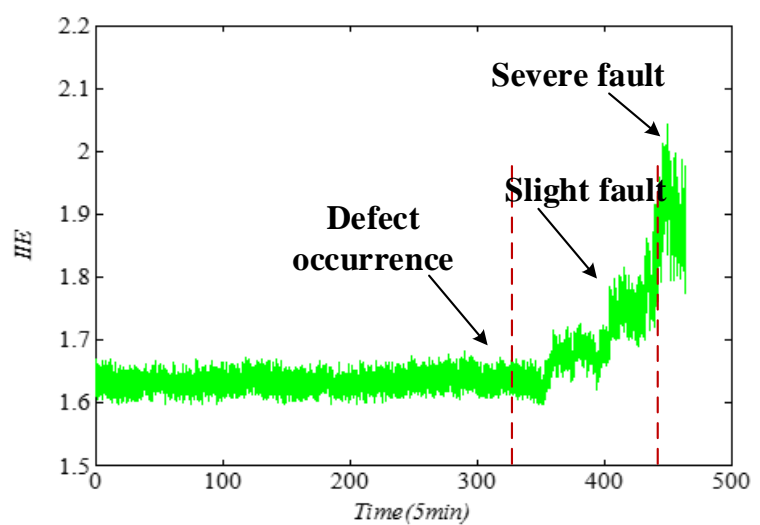

(b)

Figure 10. The IIE trend charts of vibration signals from our laboratory: (a) Inner race, (b) outer race, and (c) roller element.

\section{Conclusions}

A sensitive health indicator extraction is crucial for health monitoring and timely maintenance of bearings. This study presents a sensitive feature extraction method based on IIE and applies this method to the health evaluation of different fault bearings. We define the characteristic parameter of IIE by calculating the probability mass function and Shannon entropy of a set of time series. This parameter can effectively distinguish between typical signals and is quite sensitive to signal mutation. Thus, the IIE feature is extracted to conduct the bearing fault diagnosis experiments. The experimental results with different bearing fault cases show that the proposed method can reflect the decline in bearing monotonic performance. The IIE feature extraction can provide information on the entire process of failure occurring and gradually worsening during the operation period of the bearing. The proposed prediction method of bearing performance degradation not only can select few parameters but also can quickly detect the variation in weak signals. Therefore, the method is effective for evaluating the performance degradation of bearings.

Author Contributions: Data curation, H.Z., P.Z. and L.X.; Funding acquisition, B.J., Y.L. and G.L.; Methodology, B.J. and H.Z.; Project administration, Y.L.; Resources, L.X.; Supervision, Y.L. and G.L.; Validation, D.P.; Writing—original draft, H.Z.; Writing—review \& editing, B.J.

Funding: This work is supported in part by the National Natural Science Foundation of China (51675001, 51707001, 51605002, and 51705001), the State Key Program of National Natural Science of China (51637001), and the Natural Science Foundation of Anhui Province (1808085QE121 and 1808085QF212).

Acknowledgments: The authors are grateful to the Intelligent Maintenance System Center for providing free-bearing data download and to the anonymous reviewers for their valuable comments and suggestions.

Conflicts of Interest: The authors declare no conflict of interest. 


\section{References}

1. Henao, H.; Capolino, G.A.; Fernandez-Cabanas, M.; Filippetti, F.; Bruzzese, C.; Strangas, E.; Pusca, R.; Estima, J.; Riera-Guasp, M.; Hedayati-Kia, S. Trends in fault diagnosis for electrical machines: A review of diagnostic techniques. IEEE Ind. Electron. Mag. 2014, 8, 31-42. [CrossRef]

2. Frosini, L.; Harlişca, C.; Szabó, L. Induction machine bearing fault detection by means of statistical processing of the stray flux measurement. IEEE Trans. Ind. Electron. 2015, 62, 1846-1854. [CrossRef]

3. Kui, L.I.; Fan, Y.; Jiande, W.U. Research on bearing fault intelligent diagnosis method based on mrsvd and vpmcd. Comput. Eng. Appl. 2016, 52, 153-157.

4. Dan, X.; Jian, C.; Automatization, S.O. Method of roller bearing fault diagnosis based on feature fusion of emd entropy. J. Aerosp. Power 2015, 30, 1149-1155.

5. Liu, Y.; He, B.; Liu, F.; Lu, S.; Zhao, Y. Feature fusion using kernel joint approximate diagonalization of eigen-matrices for rolling bearing fault identification. J. Sound Vib. 2016, 385, 389-401. [CrossRef]

6. Xiao, Q.; Li, X.R.; Du, J. Characteristic parameter extraction of rolling bearing vibration phase diagram and its application in fault diagnosis. Bearing 2010, 12, 34-37.

7. Zhou, X.; Jiang, Z.; Ma, F. Fault diagnosis of rolling bearing based on improved hht energy entropy and svm. J. Mech. Transm. 2016, 12, 164-168.

8. Pandarakone, S.E.; Mizuno, Y.; Nakamura, H. Distinct fault analysis of induction motor bearing using frequency spectrum determination and support vector machine. IEEE Trans. Ind. Appl. 2017, 53, 3049-3056. [CrossRef]

9. Zhang, L.; Zhang, C.; Ji, J. Approach for bearing fault diagnosis based on kpca and elm. J. Electron. Meas. Instrum. 2018, 2, 23-29.

10. Ding, R.; Li, J. Nonlinear finite-time lyapunov exponent and predictability. Phys. Lett. A 2007, 364, 396-400. [CrossRef]

11. Muruganatham, B.; Jayakumar, T. Detection of faulty ball bearing using symbolic dynamics. Int. J. Cond. Monit. 2013, 3, 23-34. [CrossRef]

12. Chen, W.J.; Wu, J.Q. Application of hilbert-huang transform in wind turbine's mainshaft bearing fault diagnosis. Bearing 2013, 6, 59-62.

13. Hu, A.; Xiang, L.; Zhang, Y. Experimental study on the intrawave frequency modulation characteristic of rotor rub and crack fault. Mech. Syst. Signal Process. 2019, 118, 209-225. [CrossRef]

14. Basaran, C.; Yan, C.Y. A Thermodynamic Framework for Damage Mechanics of Solder Joints. J. Electron. Packag. 1998, 120, 379-384. [CrossRef]

15. Basaran, C.; Nie, S. An irreversible thermodynamics theory for damage mechanics of solids. Int. J. Damage Mech. 2016, 13, 205-223. [CrossRef]

16. Sosnovskiy, L.; Sherbakov, S. Mechanothermodynamic entropy and analysis of damage state of complex systems. Entropy 2016, 18, 268. [CrossRef]

17. Yan, R.; Gao, R.X. Approximate entropy as a diagnostic tool for machine health monitoring. Mech. Syst. Signal Process. 2007, 21, 824-839. [CrossRef]

18. Cheng, J.S.; Xing-Wei, M.A.; Yu, Y. Rolling bearing fault diagnosis method based on permutation entropy and vpmcd. J. Vib. Shock 2014, 34, 802-806.

19. Zhang, L.; Xiong, G.; Liu, H.; Zou, H.; Guo, W. Bearing fault diagnosis using multi-scale entropy and adaptive neuro-fuzzy inference. Expert Syst. Appl. 2010, 37, 6077-6085. [CrossRef]

20. Costa, M.; Goldberger, A.L.; Peng, C.K. Multiscale entropy analysis of complex physiologic time series. Phys. Rev. Lett. 2007, 89, 705-708. [CrossRef]

21. Sheng, J.L.; Zhou, M.S.; Guo, Z.P.; Liu, Z. Fault diagnosis for transformer based on fuzzy entropy. Mar. Electr. Electron. Eng. 2008, 759-762.

22. Zhao, Z.H.; Yang, S.P. Sample entropy-based roller bearing fault diagnosis method. J. Vib. Shock 2012, 31, 136-140.

23. Bandt, C.; Pompe, B. Permutation entropy: A natural complexity measure for time series. Phys. Rev. Lett. 2002, 88, 174102. [CrossRef]

24. Feng, F.; Rao, G.; Jiang, P.; Si, A. Research on Early Fault Diagnosis for Rolling Bearing Based on Permutation Entropy Algorithm. In Proceedings of the IEEE 2012 Prognostics and System Health Management Conference, Beijing, China, 23-25 May 2012; pp. 1-5. 
25. Frank, B.; Pompe, B.; Schneider, U.; Hoyer, D. Permutation entropy improves fetal behavioural state classification based on heart rate analysis from biomagnetic recordings in near term fetuses. Med. Biol. Eng. Comput. 2006, 44, 179-187. [CrossRef]

26. Feng, F. Early fault diagnosis technology for bearing based on wavelet correlation permutation entropy. J. Mech. Eng. 2012, 48, 73-79. [CrossRef]

27. Zhao, H.; Sun, M.; Deng, W.; Yang, X. A new feature extraction method based on eemd and multi-scale fuzzy entropy for motor bearing. Entropy 2016, 19, 14. [CrossRef]

28. Wu, S.-D.; Wu, C.-W.; Wu, P.-H.; Ding, J.-J. Bearing fault diagnosis based on multiscale permutation entropy and support vector machine. Entropy 2012, 14, 2650-2654. [CrossRef]

29. Sheng, S.; Zhang, L.; Gao, R.X. A systematic sensor-placement strategy for enhanced defect detection in rolling bearings. IEEE Sens. J. 2006, 6, 1346-1354. [CrossRef]

30. Shao, Y.; Di, L.U.; Yang, G.X. Application of fractional fourier transform in fault diagnostics of rolling bearing. J. Harbin Univ. Sci. Technol. 2017, 3, 68-72.

31. Ding, X.W.; Liu, B.; Liu, J.Z.; Wang, C.G.; Riemenscheider, S.D.; Hu, X.Y. Fault diagnosis of freight car rolling element bearings with adaptive short-time fourier transform. China Railw. Sci. 2005, 26, 24-27.

32. Katoh, K.; Misawa, K.; Kuma, K.; Miyata, T. Mafft: A novel method for rapid multiple sequence alignment based on fast fourier transform. Nucleic Acids Res. 2002, 30, 3059-3066. [CrossRef] [PubMed]

33. Ke, D.G.; Tong, Q.Y. Easily adaptable complexity measure for finite time series. Phys. Rev. E 2008, 77, 066215. [CrossRef]

34. Schreiber, T. Detecting and analyzing nonstationarity in a time series using nonlinear cross predictions. Phys. Rev. Lett. 1997, 78, 843-846. [CrossRef]

35. Lu, S.; Liu, F.; Yang, H.; Li, W.; Zhou, R. Transient Signal Period Detection for Bearing Fault Diagnosis Using Amplitude Demodulation and Signal Segmentation and Labeling. In Proceedings of the 11th IEEE Conference on Industrial Electronics and Applications, Hefei, China, 5-7 June 2016; pp. 1389-1393.

36. Meng, Z.; Zhan, X.; Li, J.; Pan, Z. An enhancement denoising autoencoder for rolling bearing fault diagnosis. Measurement 2018, 130, 448-454. [CrossRef]

37. Yan, R.; Liu, Y.; Gao, R.X. Permutation entropy: A nonlinear statistical measure for status characterization of rotary machines. Mech. Syst. Signal Process. 2012, 29, 474-484. [CrossRef]

38. National Aeronautics and Space Administration. Available online: http://ti.Arc.Nasa.Gov/tech/dash/ pcoe/prognostic-data-repository/\#bearing (accessed on 28 July 2015).

39. Qiu, H.; Lee, J.; Lin, J.; Yu, G. Wavelet filter-based weak signature detection method and its application on rolling element bearing prognostics. J. Sound Vib. 2006, 289, 1066-1090. [CrossRef] 